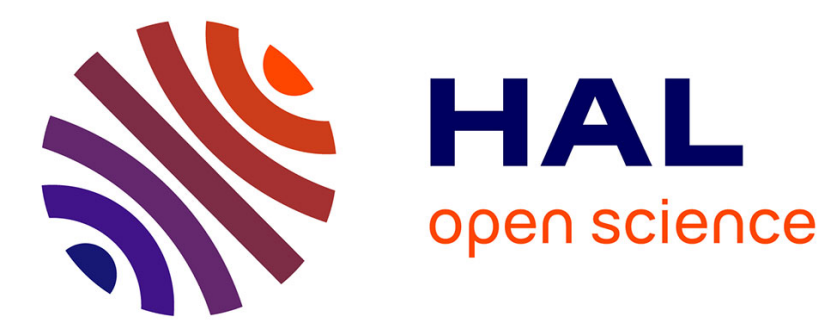

\title{
Vingt ans de réformes des systèmes éducatifs dans les pays en développement
}

Georges Solaux

\section{To cite this version:}

Georges Solaux. Vingt ans de réformes des systèmes éducatifs dans les pays en développement. Politiques et Management public, 2005, 23 (1), pp.151-158. halshs-00004944

\section{HAL Id: halshs-00004944 https://shs.hal.science/halshs-00004944}

Submitted on 31 Jan 2019

HAL is a multi-disciplinary open access archive for the deposit and dissemination of scientific research documents, whether they are published or not. The documents may come from teaching and research institutions in France or abroad, or from public or private research centers.
L'archive ouverte pluridisciplinaire HAL, est destinée au dépôt et à la diffusion de documents scientifiques de niveau recherche, publiés ou non, émanant des établissements d'enseignement et de recherche français ou étrangers, des laboratoires publics ou privés. 


\title{
Vingt ans de réformes des systèmes éducatifs dans les pays en développement Georges Solaux (IREDU, Université de Bourgogne)
}

\author{
Publié dans Politiques et management public, vol. $23 n^{\circ}$ 1, 2005. pp. 151-158.
}

Résumé :

La situation politique, économique et sociale des pays en développement est difficile alors que pratiquement tous, depuis maintenant plus de vingt ans, ont mis en œuvre des réformes très importantes élaborées en fonction des plans d'ajustement structurel initiés par le FMI, et financées par des projets soutenus par les grands bailleurs de fonds internationaux (multilatéraux ou bilatéraux). Parmi ces pays, cinquante et un ont été identifiés par la Banque mondiale comme présentant un niveau de développement tellement faible, associé à un taux d'endettement tellement élevé, qu'ils sont susceptibles de bénéficier de l'initiative dite PPTE (Pays Pauvres Très Endettés). L'objectif prioritaire de l'initiative PPTE concerne la réduction de la pauvreté. Dans ce cadre l'école paraît instrumentalisée dans une perspective qui peut sembler étroitement utilitariste, car la finalité est d'abord centrée sur l'efficacité externe de celle-ci. Nous tenterons cependant, au cours des quatre chapitres du texte qui suit, d'appréhender les marges de liberté dont disposent les gouvernements en place au regard des exigences des grands bailleurs de fonds. S'agit-il de politiques publiques sous influence ? Y a-t-il limitation des initiatives, déresponsabilisation du politique par exportation de pensée unique émanant des pays les plus industrialisés?

La génération de réformes des politiques publiques étudiée dans cet article concerne le secteur éducatif des pays en développement et notamment les pays d'Afrique subsaharienne dont le Centre d'Etudes Prospectives et d'Informations Internationales (CEPII) synthétise la situation dans ces quelques propositions : « la croissance économique de l'Afrique a légèrement reculé en 2002 (3,4\% après 3,6\% en 2001). Le FMI note que certains progrès ont été enregistrés dans le domaine des finances publiques et se traduisent notamment par un recul de l'inflation. Mais les conditions d'une croissance plus forte ne sont pas encore réunies ${ }^{1} \gg$. Ce constat sera approfondi dans le premier chapitre du texte qui suit sur la base de la consultation des bases de données internationales (FMI, Banque mondiale, UNESCO...) mises à la disposition du public et des chercheurs soit sous forme de document écrit soit par consultation en ligne ${ }^{2}$.

La situation politique, économique et sociale de ces pays est difficile alors que pratiquement tous ont mis en œuvre des réformes structurelles très importantes élaborées sur la base des plans d'ajustement structurel initiés par le FMI, et financées par des projets soutenus par les grands bailleurs de fonds internationaux (multilatéraux ou bilatéraux). L'analyse des projets et réformes sera proposée dans le second chapitre en centrant notamment l'analyse sur le secteur social et l'école. Les résultats de ces différents projets et réformes seront présentés et analysés dans le troisième chapitre soit sur la base de matériaux collectés dans les pays concernés, soit sur la base d'études réalisées par de grands organismes internationaux et disponibles pour le public. Enfin, nous envisagerons les perspectives d'avenir du management des politiques publiques dans ces pays à la lumière des projets de développement des secteurs éducatifs prévus à l'horizon 2015 en vue de parvenir à l'« Education Pour Tous» (EPT), et financés grâce à l'initiative «Pays Pauvres Très Endettés» (PPTE).

\section{Quelques caractéristiques des pays en développement et de 1'Afrique subsaharienne en particulier}

Pour mieux saisir la spécificité des pays en développement il semble nécessaire d'en passer par une opposition entre pays dits riches et pays pauvres en vue de mieux situer la place de l'Afrique subsaharienne. Le rapport « World Developpement Indicators 2003 » publié par la Banque mondiale ${ }^{3}$ montre que les disparités observées en matière de

\footnotetext{
${ }^{1}$ CEPII (2003), L'économie mondiale, la Découverte, Paris

${ }^{2}$ Exemple www.banguemondiale.org, ou encore PNUD (Programme des Nations Unies pour le Développement)

${ }^{3}$ Banque mondiale (2003), World Developpment Indicators. Site www.banquemondiale.org.
} 
niveau de vie, entre les régions du monde, sont considérables

Répartition du nombre de personnes vivant avec moins de 1 dollar parjour dans le monde (exprimé en millions)

\begin{tabular}{|l|c|c|}
\hline Régions & 1990 & 1999 \\
\hline Asie de l'Est et Pacifique & 486 & 279 \\
\hline Europe de l'Est et Asie centrale & 6 & 24 \\
\hline Amérique latine et Caraïbes & 48 & 57 \\
\hline Moyen Orient et Afrique du Nord & 5 & 6 \\
\hline Asie du Sud & 506 & 488 \\
\hline Afrique subsaharienne & 241 & 315 \\
\hline Total & 1292 & 1169 \\
\hline
\end{tabular}

Le Programme des Nations Unies pour le Développement (PNUD) publie tous les ans les « Indicateurs du développement humain» ${ }^{4}$. L'indicateur de développement humain chiffre le niveau atteint par chaque pays dans trois domaines: espérance de vie, niveau d'accès au savoir appréhendé par le taux d'alphabétisation des adultes et le taux brut de scolarisation tous niveaux confondus, et enfin le PIB par habitant exprimé en Parités de Pouvoir d'Achat (PPA). La composition des trois indicateurs ${ }^{5}$ permet d'obtenir un indicateur synthétique de développement humain (IDH) dont la valeur la plus élevée est 1 et la plus faible 0.

Indicateurs de développement humain PNUD 2001

\begin{tabular}{|l|c|c|c|c|c|}
\hline \multicolumn{1}{|c|}{ Régions } & $\begin{array}{c}\text { Espérance } \\
\text { de vie }\end{array}$ & $\begin{array}{c}\text { Alphabétisation } \\
\text { des adultes }\end{array}$ & $\begin{array}{c}\text { Tx de scol } \\
\text { Tous nivx }\end{array}$ & $\begin{array}{c}\text { PIB/tête } \\
\text { En PPA }\end{array}$ & IDH \\
\hline Pays en développement & 64,5 & 72,9 & 61 & 3350 & 0,647 \\
\hline . les moins avancés & 51,7 & 51,6 & 38 & 1178 & 0,442 \\
\hline . Afrique subsaharienne & 48,8 & 59,6 & 4 & 1640 & 0,467 \\
\hline OCDE à revenu élevé & 78 & 100 & 94 & 26050 & 0,928 \\
\hline Monde & 66,7 & & 65 & 6980 & 0,716 \\
\hline
\end{tabular}

Les très faibles performances de l'Afrique subsaharienne trouvent une part de leur explication dans une organisation économique et sociale qui présente des caractéristiques communes : un taux de croissance élevé de la démographie, des inégalités hommes-femmes et garçons-filles, une population vivant essentiellement en milieu rural mais dont l'exode vers les grands centres urbains provoque une surpopulation sans que les conditions sanitaires élémentaires soient toujours assurées, une relative instabilité politique, des recettes fiscales faibles, des performances en matière de scolarisation et de lutte contre les grandes épidémies faibles. Ces éléments relèvent d'une économie informelle dominante. Au niveau éducatif les taux de scolarisation sont peu élevés, la combinaison de taux de redoublement et d'abandons élevés provoque une efficacité interne faible ; les différences de scolarisation observées entre le milieu rural et le milieu urbain, entre les filles et les garçons montrent une organisation inéquitable.

Pour remédier à cette situation quel a été l'ordre économique proposé par les bailleurs de fonds internationaux aux pays en développement au cours des dernières décennies ?

\section{Une génération de réformes}

A la suite des indépendances et jusqu'au milieu des années soixante-dix le "mythe de l'Etat développeur" a précédé le

\footnotetext{
${ }^{4}$ http://www.undp.org/hdr2001/french/

${ }^{5}$ Voir Rapport sur le Développement Humain, page 238
} 
mythe de "l'Etat fantoche" 6 et ce n'est qu'au cours des vingt-cinq dernières années que les pressions destinées à limiter la rôle de l'Etat et à "privatiser" se sont développées. "La Théorie économique du développement des années cinquante-soixante s'est dans l'ensemble communément articulée autour d'une vision assignant aux Etats du Tiers monde une capacité à produire via une modernisation industrielle accélérée, un développement économique et social prométhéen ${ }^{7}$. Cette vision dominante, dans les pratiques et les approches scientifiques fit progressivement place à une pensée économique fondant le développement sur une limitation du rôle des Etats : « Les politiques font désormais volte-face et adoptent l'idée que la privatisation doit s'étendre à des activités aussi stratégiques que les services publics qui conditionnent le développement à long terme ${ }^{8} »$.

Les plans d'ajustement structurel imposés par le FMI et les projets de la Banque mondiale se situent dans cette condamnation de l'Etat et dans la promotion de la privatisation des entreprises publiques et de pans du secteur public non marchand. Ces plans ont pour objectif commun et pour finalité macroéconomique "la reprise de la croissance dans l'équilibre de la balance des paiements" ${ }^{2}$. Selon le FMI cet objectif peut être atteint si :

-un accroissement de la déréglementation est installé (voir l'ouverture actuelle des frontières promue par l'OMC ${ }^{10}$ ), -une accélération de la privatisation du secteur public est entreprise.

Depuis le début des années quatre-vingt on assiste donc à la mise en place de réformes et de projets dont le fondement théorique repose sur le « moins d'Etat». L'aide apportée par le FMI est suspendue au respect de ces principes et conditionne par conséquent les choix effectués par les gouvernements en place. De nombreuses conférences internationales ont rassemblé les pays en développement en vue d'harmonier la mise en œuvre de ces pratiques ${ }^{11}$. Les pays en développement ont massivement adhéré à ces grands objectifs généraux et en ont fait le fil conducteur des réformes entreprises depuis 1990 .

Concernant le secteur éducatif, et se fondant sur la notion de taux de rendement privés de l'éducation (autrement dit sur les bénéfices escomptés en matière de salaires d'une scolarisation poussée), et donc sur le constat que l'Etat engage des dépenses publiques pour des bénéfices privés, les organismes internationaux conseillent aux pays, en privatisant et/ou en rendant payants les niveaux secondaire et supérieur, de dégager les budgets publics qui leur étaient consacrés pour les transférer au bénéfice du primaire et des populations qui n'ont pas accès à l'école. Au niveau de l'enseignement primaire les bénéfices publics escomptés étant plus importants que les bénéfices privés, une part de financement public est admise.

\section{Pour quels résultats}

Ces décennies de projets et de réformes n'ont pas permis d'atteindre les objectifs escomptés. Au niveau mondial la reprise à Dakar (en 2000) d'objectifs formulés 10 ans plus tôt à Jomtien (en 1990), et fixés en l'état pour 15 années plus tard (2015) est un cuisant aveu d'échec. La situation du système éducatif apparaît en réalité comme conséquente de la détérioration d'ensemble des Etats durant les années quatre-vingt-dix. Cette désorganisation se mesure aux désordres politiques et à la croissance de la pauvreté. Les désordres politiques génèrent dans bon nombre de pays en développement des rébellions, des coups d'Etat, des guerres civiles. Sans atteindre l'intensité de tels conflits de

\footnotetext{
${ }^{6}$ Petiteville F., (1998) "Les figures mythiques de l'Etat dans l'économie en développement", Problèmes économiques, $\mathrm{n}^{\circ} 2,587$

${ }^{7}$ Petiteville F., (1998) "Les figures mythiques de l'Etat dans l'économie en développement", Problèmes économiques

${ }^{8}$ Plane P., (1998) «Les services publics africains à l'heure du désengagement de l'Etat», Problèmes économiques, $n^{\circ} 2$, 587

9 Plane P, (1994), "Les fondements théoriques des politiques de privatisation dans les PED", Revue Française d'Economie

${ }^{10}$ Organisation Mondiale du Commerce

11 La conférence mondiale sur l'Education Pour Tous (EPT) organisée à Jomtien en 1990 par I'UNESCO, la Banque mondiale, I'UNICEF. Le forum de Dakar organisé en août 2000 a reconduit les objectifs à atteindre pour 2015 . Le «sommet mondial pour le développement durable» qui s'est tenu à Johanesbourg en septembre 2002. Enfin, le programme «La décennie de l'alphabétisation des Nations Unies: Education Pour Tous 2003-2012 »
} 
société, des pays comme Haïti ou Madagascar ont connu durant cette décennie des soubresauts graves qui ont obéré leur développement.

La situation en Haïti peut être résumée dans l'extrait suivant: «depuis la restauration d'un Etat légitime en 1994 les efforts du Gouvernement, soutenu en ce sens par la communauté des bailleurs de fonds, visent à développer et consolider un Etat de Droit basé sur le respect des droits de l'homme et des libertés fondamentales ... A cette même époque (1994), la vie du pays a été marquée par une série de crises politiques menant à une situation de blocage en juin 1997 (démission de t'exécutif, une partie des ministres, dont le Premier Ministre, ayant cessé en octobre d'assurer la gestion des affaires courantes ; parlement ayant cessé ses activités législatives depuis)... Les raisons majeures sont tout d'abord la lutte des partis politiques pour le pouvoir mais aussi les divergences dans la vision du développement souhaitable pour le pays. L'un des facteurs en cause est en effet l'opposition d'une partie du Parlement au programme de réformes macroéconomiques entamé par le Gouvernement avec le soutien des bailleurs de fonds, et qui fut concrétisé en 1996 par la signature avec le FMI d'un programme d'ajustement structurel ${ }^{12}$ ». Ces éléments conjugués à la corruption, à l'utilisation malavisée des crédits publics provoquent une paralysie économique du pays et une intensification des problèmes sociaux avec en particulier la progression de la pauvreté, un rendement très faible du secteur éducatif alors que la population est très «demandeuse» de scolarisation pour ses enfants ${ }^{13}$. L'exemple de Madagascar est proche de celui d'Haïti quant à la pauvreté de la population mais moins profond sur le plan politique. Dans le champ du secteur éducatif, les problèmes politiques et sociaux et les difficultés budgétaires ont cependant contribué à générer un système éducatif peu efficace et inéquitable ${ }^{14}$.

Si tous les pays en développement ne connaissent pas des problèmes politiques et sociaux aussi difficiles à résoudre, il en est néanmoins cinquante et un qui ont été identifiés par la Banque mondiale comme présentant un niveau de développement tellement faible, associé à un taux d'endettement tellement élevé, qu'ils sont susceptibles de bénéficier de l'initiative dite PPTE (Pays Pauvres Très Endettés).

\section{Quelles perspectives?}

L'initiative PPTE a été lancée par le FMI et la Banque mondiale en 1996, relayée par le G8 ${ }^{15}$, puis appuyée par 180 pays. Cette initiative est destinée à aider les pays pauvres et les plus endettés à réduire leur dette et à mettre en place une stratégie de réduction de la pauvreté ${ }^{16}$. Sous sa forme actuelle l'initiative vise donc à lier réduction de la dette et réduction de la pauvreté en faisant de sorte que la dette n'empêche pas les pays d'investir pour l'avenir. Le mécanisme destiné à sélectionner les pays qui pourront bénéficier de l'initiative est relativement proche des méthodes développées par les grands bailleurs de fonds lorsqu'ils évaluent un projet ou un prêt. Le pays doit présenter un plan de développement à l'horizon 2015 pour le secteur concerné (santé, éducation, petite enfance, éducation non formelle ...). Ce projet doit comporter les objectifs à atteindre, les indicateurs de résultats attendus mais aussi un cadrage macroéconomique qui précise l'évolution attendue du PIB, les recettes fiscales, la part qui sera consacrée à l'éducation et à l'enseignement primaire, les objectifs de scolarisation, le salaire des enseignants ... Des conseils sont alors donnés au pays pour revoir tel ou tel indicateur en fonction de son niveau supposé de performance et de ses capacités de financement. Ce travail réalisé par les bailleurs de fonds internationaux auprès des pays qui souhaitent s'engager dans cette voie, s'apparente à la fois à la traditionnelle mise en place des conditionnalités du FMI que le pays ne maîtrise pas, mais aussi à du conseil formation des responsables en matière de management des organisations éducatives. Les détracteurs et défenseurs de la démarche entreprise choisiront leur camp...

Si cette approche situe l'initiative PPTE dans le cadre classique de l'intervention des bailleurs de fonds

\footnotetext{
${ }^{12}$ Dom C., Karroum F., (1998), Programme d'appui au renforcement de la gouvernance du secteur éducatif en Haïti

${ }^{13}$ A la fin des années quatre-vingt-dix, Haïti est présenté comme l'un des pays les plus pauvres du monde. Les deux tiers de la population vivent en milieu rural et $80 \%$ d'entre elle vit en dessous du seuil de pauvreté

14 Banque mondiale, "Education et formation à Madagascar. Vers une politique nouvelle pour la croissance économique et la réduction de la pauvreté», les grands rapports économiques de la Banque mondiale. (2002)

${ }^{15}$ groupe des 8 pays les plus industrialisés

${ }^{16}$ Voir www.banquemondiale.org et chercher PPTE
} 
internationaux, elle s'en éloigne par le fait que les crédits dégagés par la réduction de la dette seront directement intégrés au Budget de l'Etat, avec donc une plus grande latitude pour les pays de disposer et, comme ils l'entendent, des fonds ainsi rendus disponibles. Concernant la finalité et les objectifs généraux des plans prévus, la lecture de quelques projets tend à montrer que les choix politiques sont relativement communs aux différents pays et qu'ils présentent un certain caractère «d'utilitarisme ». C'est parce que l'objectif prioritaire concerne la réduction de la pauvreté que l'école paraît instrumentalisée dans une perspective qui peut sembler étroitement utilitariste, car la finalité est d'abord centrée sur l'efficacité externe de celle-ci. Mais si le facteur humain et plus précisément le capital humain ont un sens, il semble difficile d'échapper à la nécessité d'en passer par la formation des femmes et des hommes pour sortir, comme le précise l'un des projets, « de la trappe de la pauvreté », via le développement économique. C'est sans doute parce qu'il n'y a pas d'alternative au développement humain pour réduire la pauvreté dans ces pays que les objectifs assignés à l'école sont systématiquement extériorisés et rapportés d'abord à l'économie.

\section{Conclusion}

Alors que la génération de réformes des vingt dernières années du $20^{\text {ème }}$ siècle n'a pas été accompagnée des effets escomptés, les premières années du $21^{\text {eme }}$ sont marquées par la relance des objectifs de scolarisation pour tous en vue de lutter contre la pauvreté. Cependant, parallèlement au développement de ces projets, une résistance culturelle locale se structure pour résister à l'expansionnisme de la pensée libérale occidentale. Le travail réalisé par Aminata Traoré sur «le viol de l'imaginaire » s'inscrit dans ce cadre : « les nations riches, le FMI, la Banque mondiale et l'Organisation mondiale du commerce ont enfin pris conscience de la gravité de la situation dans l'hémisphère sud, et plus particulièrement en Afrique. Les maîtres du monde perçoivent maintenant clairement l'effet boomerang des choix macroéconomiques qu'ils imposent au reste de la planète. Leur pouvoir est d'autant plus limité que les citoyens et citoyennes du Sud savent évaluer et éventuellement dénoncer leurs actions $^{17}$ ». Quels seront donc les effets des nouvelles politiques mises en œuvre? Qu'en sera-t-il si de nouveaux échecs au développement sont constatés après l'initiative PPTE?

\section{BIBLIOGRAPHIE}

BRUMS, MINGAT, RAKOTOMALALA, (2003), Réaliser la scolarisation universelle en 2015. une chance pour tous les enfants du monde. World Bank, Washington.

CARROUE L. (2002). Géographie de la mondialisation, Armand Colin, Paris.

HIBOU B, (1998), «Banque mondiale: les méfaits du catéchisme économique». Esprit, Août Septembre 1998.

SOLAUX G.,(2003) «L'évaluation externe, les arbitrages entre efficacité et équité». Colloque ADMEE, Liège 2003.

TRAORE A. (2002), Le viol de l'imaginaire, Actes Sud Fayard, Paris.

${ }^{17}$ Traoré A, (2002), Le viol de l'imaginaire, Actes Sud Fayard, Paris, page 200 